\title{
miR-6852 serves as a prognostic biomarker in colorectal cancer and inhibits tumor growth and metastasis by targeting TCF7
}

\author{
BAO-HONG CUI ${ }^{1}$ and $\mathrm{XUAN} \mathrm{HONG}^{2}$ \\ Departments of ${ }^{1}$ Clinical Laboratory and ${ }^{2}$ Thoracic Medical Oncology, \\ Harbin Medical University Cancer Hospital, Harbin, Heilongjiang 150086, P.R. China \\ Received February 15, 2018; Accepted May 25, 2018
}

DOI: $10.3892 /$ etm.2018.6259

\begin{abstract}
MicroRNAs (miRs) are have been demonstrated to serve important functions in the genesis of human cancer, including colorectal cancer (CRC). The role of miR-6852 in $\mathrm{CRC}$ remains unknown. In this study, it was demonstrated that miR-6852 was underexpressed in CRC tissues compared with adjacent normal tissues. Moreover, the expression of miR-6852 was negatively correlated with CRC metastasis, whereas positively correlated with patient prognosis. It was revealed that the overexpression of miR-6852 significantly inhibited the proliferation and invasion of CRC cells. miR-6852 overexpression reduced CRC cells in the $\mathrm{S}$ phase. TCF7 was identified to be a direct target of miR-6852 in CRC cells. Overexpression of miR-6852 significantly inhibited the mRNA and protein levels of TCF7 in CRC cells. Furthermore, TCF7 was highly expressed in CRC tissues and cell lines. TCF7 expression was negatively correlated with miR-6852 levels in CRC tissues. Finally, knockdown of TCF7 significantly suppressed the proliferation and invasion of CRC cells. Taken together, the results of the present study indicated that miR-6852 serves as a tumor suppressor in CRC through targeting TCF7.
\end{abstract}

\section{Introduction}

Colorectal cancer (CRC) is one of the most prevalent and malignant cancers in the world, with more than one million new diagnosed patients each year (1). CRC contributes to a lot of cancer-related deaths. CRC progression is accompanied with accumulation of mutations, which lead to inactivation of tumor suppressors or activation of oncogenes (2). Especially, activation of $\mathrm{Wnt} / \beta$-catenin signaling pathway is observed in almost $90 \%$ of patients diagnosed with CRC $(3,4)$. Although much effort has been made, no effective therapeutic approach

Correspondence to: Professor Xuan Hong, Department of Thoracic Medical Oncology, Harbin Medical University Cancer Hospital, 150 Haping Road, Harbin, Heilongjiang 150086, P.R. China

E-mail: xuanhongtj@163.com

Key words: miR-6852, colorectal cancer, proliferation, invasion, TCF7 has been developed to fully treat CRC. The five-year survival rate of CRC patients remains quite poor (5). Therefore, there is an urgent need to explore the underlying mechanism of CRC progression.

MicroRNAs (miRNAs/miRs) are a class of naturally short noncoding RNAs with a length of 19-25 nucleotides and largely expressed in almost all cell types (6). miRNAs have been demonstrated to suppress gene expression by association with the specific sequence in the 3'-untranslated region (3'-UTR) of target mRNAs $(7,8)$. More and more evidences show that miRNAs exert important functions in a diversity of biological processes, and influence cell fate and functions (9-11). So far, a large amount of miRNAs have been shown to serve as tumor suppressors or oncogenes in almost all types of cancers, including lung cancer, breast cancer, gastric cancer and CRC (12-16). For example, microRNA-381 inhibits the metastasis of gastric cancer by targeting TMEM16A expression (17). Downregulation of miR-218 contributes to epithelial-mesenchymal transition and tumor metastasis in lung cancer by targeting Slug/ZEB2 signaling (18). However, the roles of most miRNAs in CRC still remain largely unknown.

miR-6852 has been shown to induce cell cycle arrest and necrosis in cervical cancer cells (19). Whether miR-6852 regulates CRC progression requires to be investigated. In this study, we found that miR-6852 was downregulated in CRC tissues and correlated with tumor metastasis and patients' prognosis. Moreover, we showed that overexpression of miR-6852 significantly inhibited the proliferation and invasion of CRC cells. Mechanistically, we found that TCF7 is a direct target of miR-6852. We also demonstrated that TCF7 was upregulated in CRC tissues, and promoted CRC cell proliferation and invasion. Taken together, our study demonstrated that miR-6852 acts as a tumor suppressor in CRC through targeting TCF7.

\section{Materials and methods}

Patient samples. The Ethics Committee of Harbin Medical University Cancer Hospital (Harbin, China) approved this study, and all patients gave their informed consent prior to surgery. Forty-four CRC tissues and 44 adjacent normal tissues from human patients were obtained from Harbin Medical University Cancer Hospital. The clinicopathological data of the patients were listed in Table I. For mRNA extraction, samples were 
frozen in liquid nitrogen immediately after surgical removal and maintained at $-80^{\circ} \mathrm{C}$ until use. Additional samples were fixed in $10 \%$ neutral-buffered formalin overnight, processed, paraffin embedded, and sectioned.

Cell lines and cell culture. Human CRC cancer cell lines (SW480, HCT8, HT29, LOVO and HCT116) and normal colorectal mucosa cells cell line (FHC) were obtained from ATCC (Manassas, VA, USA) and maintained in DMEM medium supplemented with $10 \%$ fetal bovine serum (FBS; Invitrogen; Thermo Fisher Scientific, Inc., Waltham, MA, USA), $100 \mathrm{U} / \mathrm{ml}$ penicillin, and $100 \mathrm{mg} / \mathrm{ml}$ streptomycin (Invitrogen; Thermo Fisher Scientific, Inc.). Cells were incubated at $37^{\circ} \mathrm{C}$ in a humidified atmosphere with $5 \% \mathrm{CO}_{2}$.

Cell transfection. siRNA against TCF7 (5'-GGAAGAGAG GACAAGGAAT-3') and miR-6852 mimics (5'-CCCUGG GGUUCUGAGGACAUG-3'), inhibitors (5'-CATGTCCTC AGAACCCCAGGG-3') or controls (5'-UCACAACCUCCU AGAAAGAGUAGA-3') were synthetized by Invitrogen (Thermo Fisher Scientific, Inc.). 50 nM miR-6852 mimics, inhibitors or controls were transduced into HCT8 and SW480 cells with Lipofectamine 3000 (Invitrogen; Thermo Fisher Scientific, Inc.) at $20^{\circ} \mathrm{C} .48 \mathrm{~h}$ after transfection, the culture medium was replaced with fresh medium, followed by analysis.

Cell proliferation. Each well of a 96-well plate contained approximately 5,000 transfected cells. Cell viability was assessed using a Cell Counting Kit-8 (CCK-8) assay (7 Sea Biotech, Shanghai, China). Following incubation for $72 \mathrm{~h}, 10 \mu \mathrm{l}$ CCK-8 was added to each well and incubated for $2 \mathrm{~h}$ in an incubator. The absorbance value was determined using a multimode microplate reader (Berthold Technologies GmbH \& Co. KG, Bad Wildbad Germany) at 450 nm.

Flow cytometry. Cells transiently transfected with the indicated plasmids were harvested $48 \mathrm{~h}$ after transfection by trypsinization, washed with ice-cold phosphate-buffered saline, and fixed with $70 \%$ ethanol overnight. The cells were then collected by centrifugation and resuspended in PI solution $[50 \mathrm{mg} / \mathrm{ml}$ in phosphate-buffered saline (PBS)] containing $0.25 \mathrm{mg} / \mathrm{ml}$ of RNase A. After incubation for $15 \mathrm{~min}$ in the dark at $4^{\circ} \mathrm{C}$, the cells were analyzed by flow cytometry (FACS Canto II; BD Biosciences, Franklin Lakes, NJ, USA) using an instrument equipped with Cell Quest software (BD Biosciences). The percentages of the cells in $\mathrm{S}$ phase were counted and compared.

Transwell assay. Before experiment, the Matrigel was melted at $4^{\circ} \mathrm{C}$ and diluted with serum-free culture medium (1:3). On the surface of polycarbonate membrane, $40 \mu$ l diluted matrigel was spread and placed in the incubator for $4 \mathrm{~h}$ of coagulation for later use. After cells in logarithmic phase were starved in the serum-free DMEM media for $24 \mathrm{~h}$, cells were digested using $0.25 \%$ ethylene diamine tetraacetic acid (EDTA) trypsin for preparation of single-cell suspension using the serum-free DMEM culture medium, in which cell density was adjusted to $4 \times 10^{5} / \mathrm{ml}$. In the upper transwell chamber, $200 \mu 1$ serum-free cell suspension was added, and the wells were grouped according to the experiment requirement with 3 replicative
Table I. Associations between miR-6852 expression and clinical characteristics in CRC patients $(n=44)$.

\begin{tabular}{lccc}
\hline Clinicopathologic & $\begin{array}{c}\text { Low } \\
\text { expression } \\
\text { parameters }\end{array}$ & $\begin{array}{c}\text { High } \\
\text { expression } \\
(\mathrm{n}=22)\end{array}$ & P-value \\
\hline Age (years) & 10 & 7 & 0.5365 \\
$\quad$ 65 & 12 & 15 & \\
$>65$ & & & 0.5434 \\
Sex & 14 & 11 & \\
Male & 8 & 11 & \\
Female & & & 0.0268 \\
Tumor size (cm) & 4 & 12 & \\
$\leq 5$ & 18 & 10 & \\
$>5$ & & & 0.0122 \\
Lymph node metastasis & 13 & 4 & \\
Yes & 9 & 18 & \\
No & & & 0.0329 \\
TNM stage & 8 & 16 & \\
I-II & 14 & 6 & \\
III-IV & & & \\
\end{tabular}

Pearson's Chi-square tests were used. Results were considered statistically significant at $\mathrm{P}<0.05$. CRC, colorectal cancer; TNM, tumor node metastasis.

wells in each group. In the lower transwell chamber, $600 \mu 1$ DMEM medium supplemented with $10 \%$ fetal bovine serum were added into each well for $24 \mathrm{~h}$ of culture in an incubator. The chambers were taken out and washed with PBS to remove the medium. In the upper chamber, cells that failed to pass through the membrane were scrubbed using a wet cotton swab followed by $20 \mathrm{~min}$ of fixation in methanol drying at room temperature. Furthermore, cells were stained using crystal violet for $20 \mathrm{~min}$, and placed under the inverted microscope to observe the quantity of cells that passed through the membrane. Cell count was performed in 5 high magnification vision (x400), and the average cell count was used as the result.

Reverse transcription-quantitative polymerase chain reaction (RT-qPCR). Total RNAs were extracted with TRIzol according to the manufacturer's protocol. cDNA was synthesized with the M-MLV reverse transcriptase (Promega Corporation, Madison, WI, USA). Transcripts were analyzed with ABI 7300 qPCR system using specific primer pairs. Relative expressions were calculated and normalized to endogenous Actb or U6. Expressions were normalized to endogenous controls and calculated using relative quantification method $\left(2^{-\Delta \Delta C q}\right)(20)$. The primer sequences are as follows: Universal miRNA primer (5'-AACGAGACGACGACAGAC-3'), U6 (5'-GCAAATTCG TGAAGCGTTCCATA-3'), miR-6852 (5'-CCCTGGGGTTCT GAGGACATG-3'), Actb (forward, 5'-CGGCGCCCTATA AAACCCA-3'; and reverse, 5'-GAGGCGTACAGGGATAGC AC-3') and TCF7 (forward, 5'-CTGGGCAAGGAAGCCATA GG-3'; reverse, 5'-TGCTGTACCTGTGTGCTCTG-3'). 

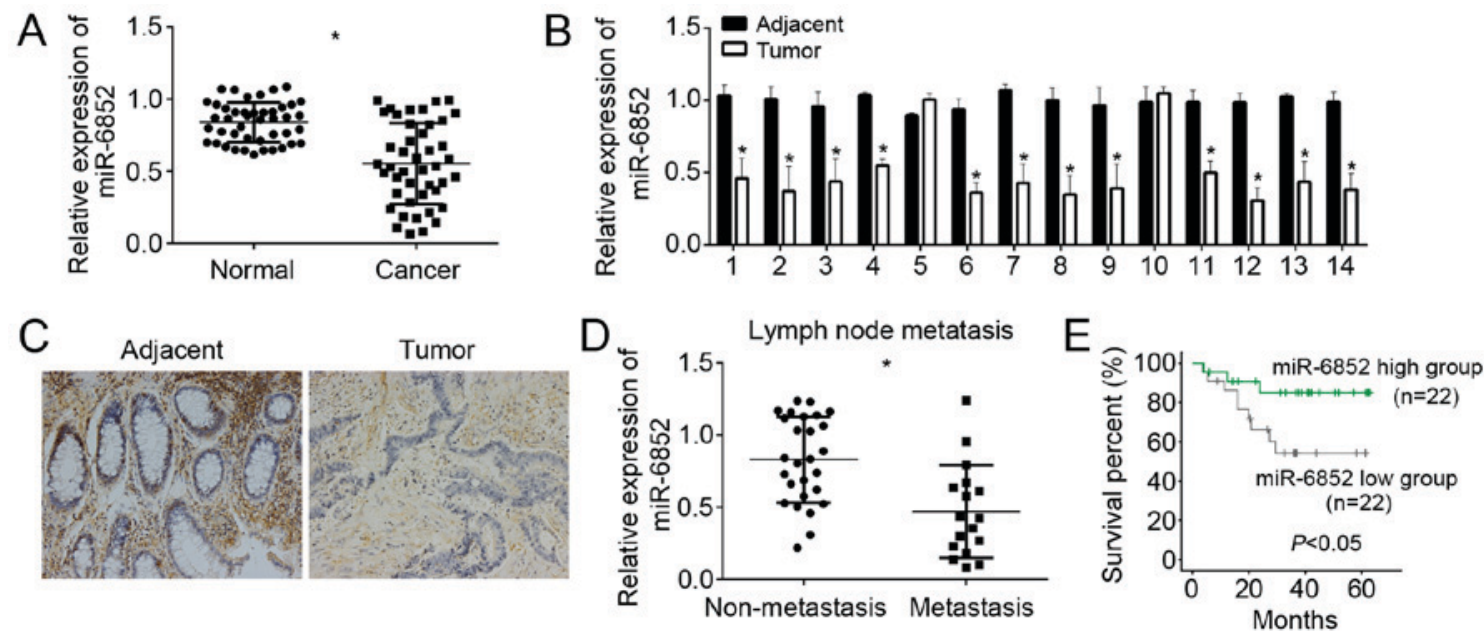

Figure 1. Expression of miR-6852 in CRC tissues and cell lines. (A) miR-6852 expression was measured in CRC tissues and adjacent normal tissues by RT-qPCR. (B) Relative expression of miR-6852 in CRC tissues and matched adjacent normal tissues by RT-qPCR. (C) Relative expression of miR-6852 in paired CRC tissues and adjacent normal tissues by RNA in situ hybridization. (D) Relative levels of miR-6852 in metastatic CRC tissues and non-metastatic CRC tissues were determined by RT-qPCR. (E) Kaplan-Meier survival curse based on miR-6852 expression in CRC tissues. Magnification, $x 100$. "P $<0.05$ vs. control group. All data presented are shown as means \pm standard deviation collected from three independent experiments. RT-qPCR, reverse transcription-quantitative polymerase chain reaction; CRC, colorectal cancer; miR, microRNA.

Western blot analysis. After transfection, CRCs total protein was extracted by RIPA buffer (Beyotime, Beijing, China). All the protein lysates were separated using 10\% SDS-PAGE and transferred onto a polyvinylidene fluoride membrane. The membrane was incubated with specific primary anti-human antibodies including TCF7 (1:1,000, cat. no. 2203; Cell Signaling Technology, Inc., Danvers, MA, USA) and GAPDH (1:5,000; Abcam, Cambridge, UK), followed by their respective appropriate secondary antibody (1:5,000; Biogot Technology, Nanjing, China). GAPDH was used as a control.

Luciferase reporter assay. The fragment of TCF7 3'-UTR containing putative miR-6852 binding sites or the mutant fragment was synthesized and inserted into the pmirGLO vector (Promega Corporation). The cells were co-transfected with these plasmids with miR-6852 mimic or miR-6852 mimic control. The Dual-Luciferase Reporter Assay System (Promega Corporation) was used to measure luciferase activity. Renilla luciferase activity was used as the normalized control.

Statistical analysis. All statistical analyses were performed using the Statistical Package for the Social Sciences version 20.0 software (SPSS, Inc., Chicago, IL, USA). Survival curves were calculated using the Kaplan-Meier method and were analyzed using the log-rank test. For comparisons, one-way analysis of variance followed by Tukey's post hoc test and two-tailed Student's t-tests were performed, as appropriate. The correlations were analyzed using Pearson's correlation coefficients. The Pearson's Chi-square test was used to assess the association between clinicopathological features and miR-6852. $\mathrm{P}<0.05$ was considered to indicate a statistically significant difference.

\section{Results}

Expression of miR-6852 in CRC tissues and cell lines. To explore the role of miR-6852 in CRC, we analyzed the expression patterns of miR-6852 in CRC tissues and adjacent normal tissues by RT-qPCR. The results showed that miR-6852 was downregulated in CRC tissues $(n=44)$ compared with adjacent normal tissues $(n=44)$ (Fig. 1A). We also checked the expression of miR-6852 in 14 pairs of CRC tissues and normal tissues and found that miR-6852 was significantly downregulated in most CRC tissues compared to matched adjacent normal tissues (Fig. 1B). Furthermore, we verified miR-6852 expression by RNA in situ hybridization. We found that miR-6852 was remarkably downregulated in CRC tissues compared with matched normal tissues (Fig. 1C). We determined the correlation between miR-6852 expression and CRC metastasis. Through RT-qPCR analysis, we found that miR-6852 displayed lower expression in CRC samples with lymph node metastasis $(n=17)$ than that without lymph node metastasis $(n=27)$ (Fig. 1D). To determine whether miR-6852 could serve as a prognostic biomarker for CRC patients, we performed Kaplan-Meier survival curse analysis according to miR-6852 expression (mean value was chosen for cut-off) in collected 44 CRC samples. We divided these samples into miR-6852 low expression group $(n=22)$ and high expression group $(\mathrm{n}=22)$. The results indicated that CRC patients with lower expression of miR-6852 showed shorter survival rate (Fig. 1E), which indicated that miR-6852 was a good prognostic biomarker.

miR-6852 overexpression suppresses CRC cell proliferation and invasion. To further investigate the effects of miR- 6852 on CRC cells, we first analyzed its expression patterns in CRC cell lines by RT-qPCR. Results indicated that miR-6852 was also downregulated in CRC cell lines compared to FHC normal cells (Fig. 2A). We chose HCT8 and SW480 cells for following experiments. We overexpressed miR-6852 by transfection with miR-6852 mimics in HCT8 and SW480 cells. RT-qPCR results showed that miR-6852 was significantly upregulated in HCT8 and SW480 cells transfected with miR-6852 mimics compared to controls (Fig. 2B). 

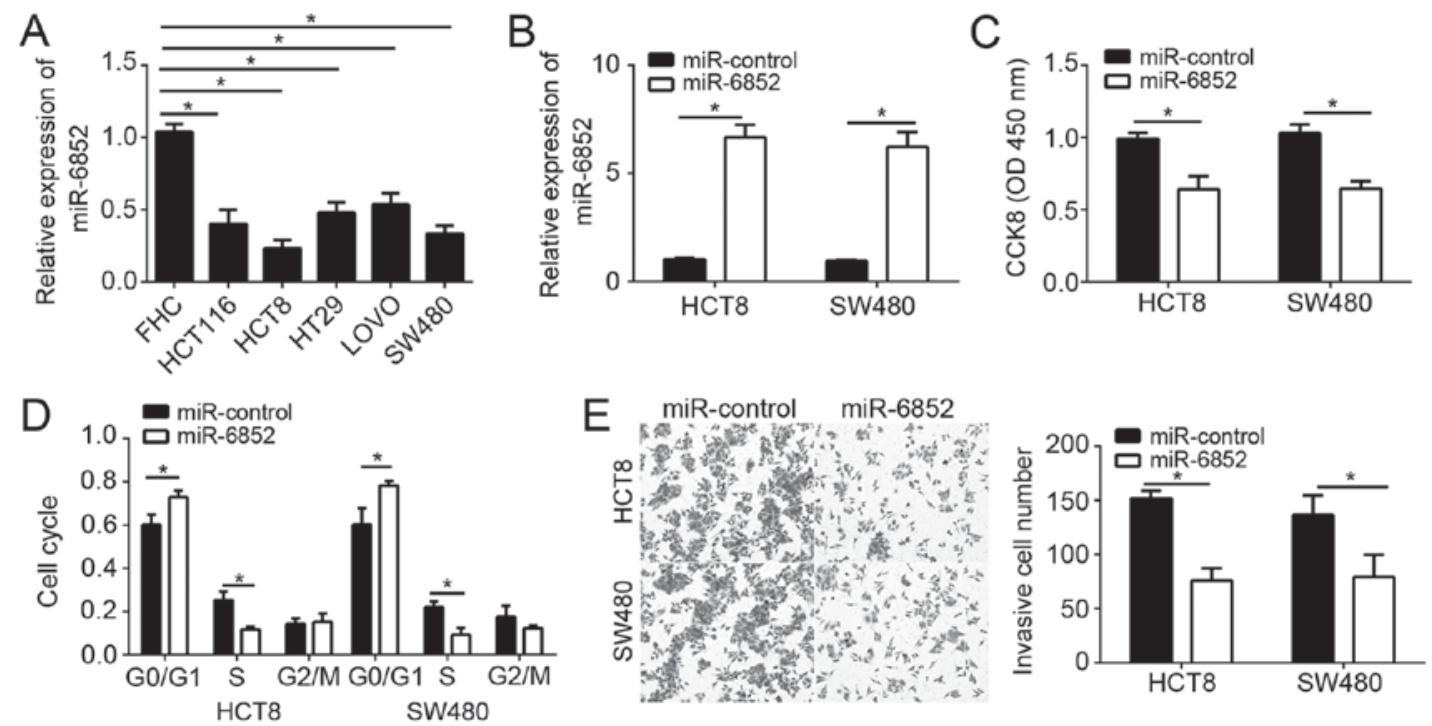

Figure 2. miR-6852 overexpression suppressed CRC cell proliferation and invasion. (A) Relative expression levels of miR-6852 in CRC cell lines and FHC cells. (B) RT-qPCR analysis for miR-6852 expression in HCT8 and SW480 cells transduced with miR-6852 mimics or control. (C) CCK-8 assay was performed to assess cell proliferation. (D) Cell percent in S phase was measured by FACS. (E) Transwell assay was used to determine the invasion of HCT8 and SW480 cells. Magnification, $x 100$. "P<0.05 vs. control group. All data were collected from three independent experiments. RT-qPCR, reverse transcription-quantitative polymerase chain reaction; CRC, colorectal cancer; miR, microRNA; CCK-8, Cell Counting Kit-8.

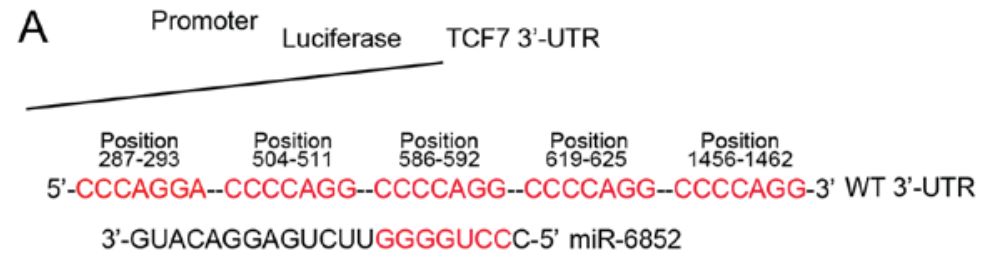

5'-GGGGUCC--GGGGUCC--GGGGUCC--GGGGUCC--GGGGUCC-3' Mut 3'-UTR
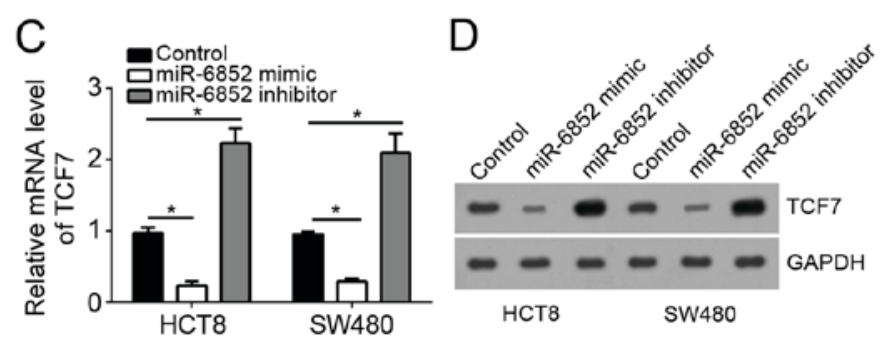

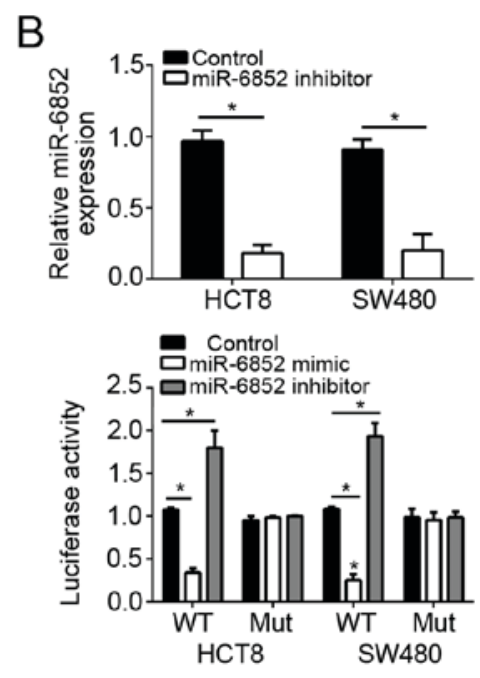

Figure 3. TCF7 was a target of miR-6852. (A) Potential binding sites of miR-6852 in the 3'-UTR region of TCF7 mRNA were shown. (B) RT-qPCR result showed miR-6852 expression was significantly repressed after transfection with inhibitors. And luciferase reporter assay indicated that overexpression of miR-6852 significantly inhibited the luciferase activity in HCT8 and SW480 cells transduced with WT-TCF7-3'-UTR, and vice versa. (C) mRNA and (D) protein levels of TCF7 in HCT8 and SW480 cells were measured by RT-qPCR and western blotting. "P<0.05 vs. control group. All data presented are shown as means \pm standard deviation collected from three independent experiments. miR, microRNA; 3'-UTR, 3'-untranslated region; RT-qPCR, reverse transcription-quantitative polymerase chain reaction.

CCK-8 assay were used to analyze cell proliferation. As shown, overexpression of miR-6852 significantly inhibited the proliferation of HCT8 and SW480 cells (Fig. 2C). What's more, FACS assay demonstrated that overexpression of miR-6852 significantly reduced the cells in S phase (Fig. 2D), indicating a decreased cell-cycle. Metastasis leads to CRC recurrence and malignance. Therefore, we analyzed the effect of miR-6852 on cell invasion. Transwell assays indicated that overexpression of miR-6852 significantly suppressed the invasion in HCT8 and SW480 cells (Fig. 2E). Taken together, these data demonstrated that miR-6852 inhibited CRC cell proliferation and invasion.
TCF7 is a target of miR-6852. To further explore the downstream mechanism, we searched for the target genes of miR-6852. By the targetscan tool, we found that TCF7 was a potential target of miR-6852. TCF7 is a key regulator of Wnt/ק-catenin signaling pathway, which is abnormally activated in most CRC patients. Therefore, we chose TCF7 for next analysis. We found that there were five potential binding sites of miR-6852 in the 3'-UTR of TCF7 mRNA (Fig. 3A). To verify it, we constructed luciferase reporter vectors. We mutated the potential binding sites in the 3'-UTR of TCF7 mRNA to construct a mutant reporter plasmid. Through luciferase reporter assays, we found that overexpression of 

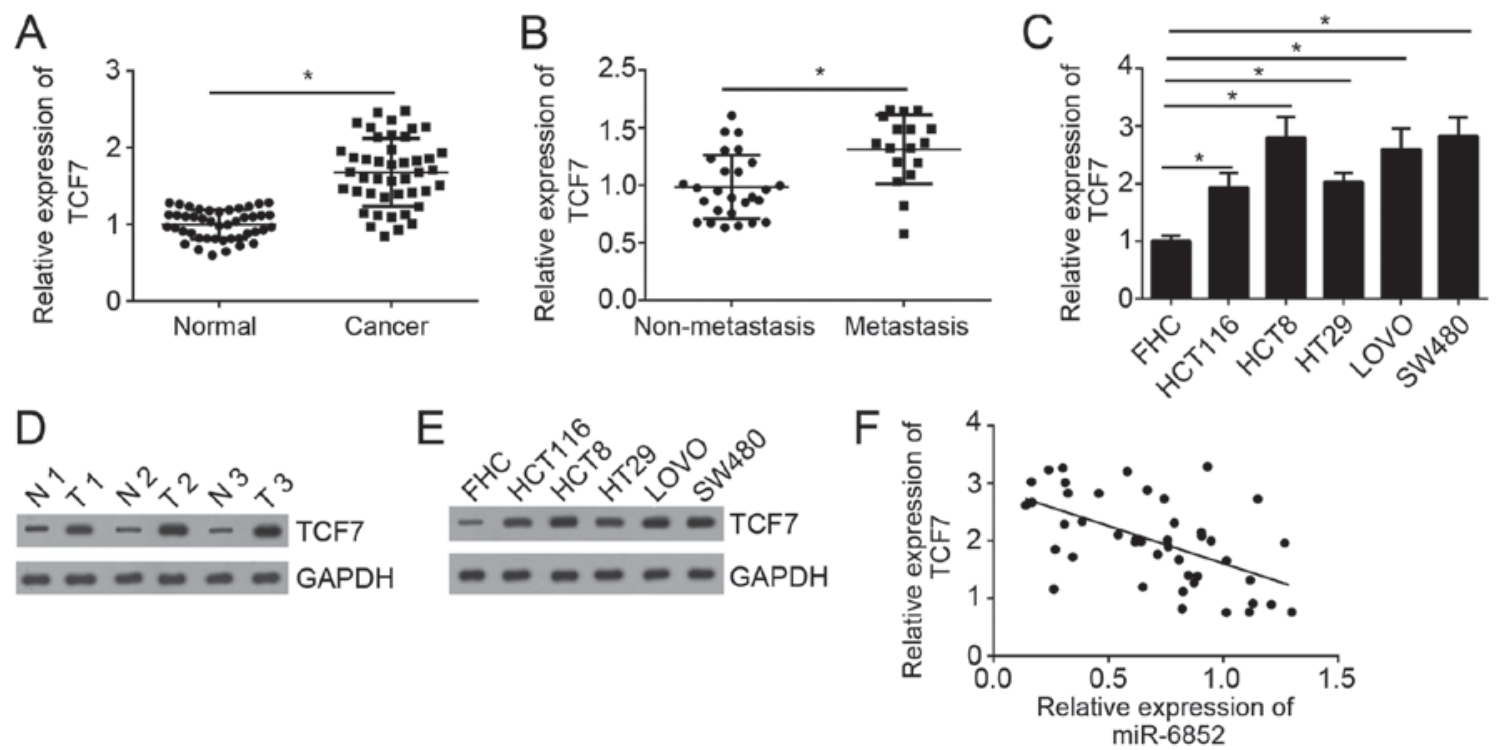

Figure 4. TCF7 was upregulated in CRC tissues. (A) Relative expression of TCF7 in CRC tissues and adjacent normal tissues by RT-qPCR. (B) Relative expression of TCF7 in metastatic and non-metastatic CRC tissues. (C) Relative expression of TCF7 in CRC cell lines. Protein levels of TCF7 in (D) CRC tissues and (E) cell lines were measured by western blotting. (F) Expression correlation between miR-6852 and TCF7 in CRC tissues. "P $<0.05$ vs. control group. All data presented are shown as means \pm standard deviation collected from three independent experiments. RT-qPCR, reverse transcription-quantitative polymerase chain reaction; CRC, colorectal cancer; miR, microRNA.

A
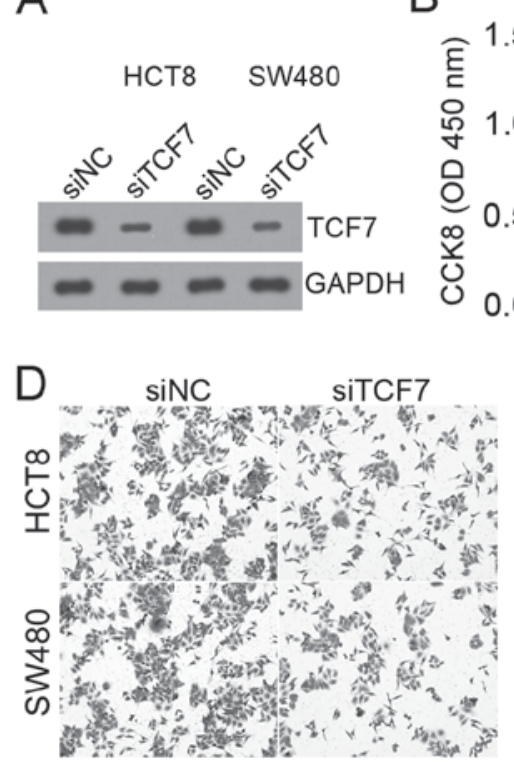

B
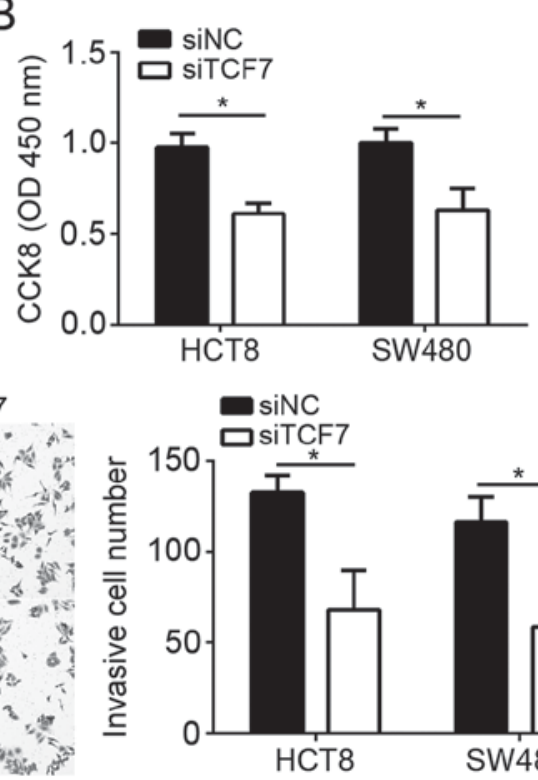

-

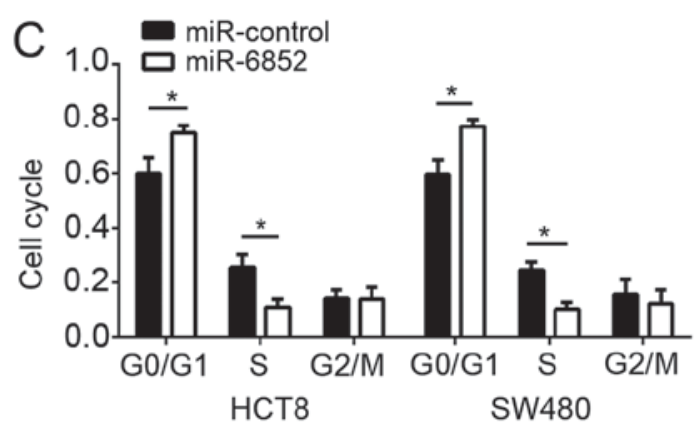

Figure 5. Knockdown of TCF7 inhibited the proliferation and invasion of CRC cells. (A) Western blotting was used to measure the protein level of TCF7 in HCT8 and SW480 cells transduced with siTCF7 or control. (B) CCK-8 assay was used for detection of cellular proliferation. (C) Cell percent in S phase was determined by FACS. (D) Cell invasion was measured by Transwell assay. Magnification, x100. "P<0.05 vs. control group. All data presented are shown as means \pm standard deviation collected from three independent experiments. CCK-8, Cell Counting Kit-8; miR, microRNA.

miR-6852 significantly inhibited the luciferase activity in HCT8 and SW480 cells transduced with WT reporter vector and vice versa, whereas transfection with mutant reporter vector had no this effect (Fig. 3B). Moreover, by RT-qPCR and western blot, we found that overexpression of miR-6852 significantly inhibited the mRNA and protein levels of TCF7 in HCT8 and SW480 cells, and vice versa (Fig. 3C and D).

TCF7 is upregulated in CRC tissues. We analyzed the expression patterns of TCF7 in CRC tissues by western blot.
As shown in Fig. 4A, we found that TCF7 expression was significantly upregulated in CRC tissues compared with adjacent normal tissues. Moreover, the expression of TCF7 was significantly upregulated in metastatic CRC tissues compared with non-metastatic tissues (Fig. 4B). Consistently, the expression of TCF7 was also upregulated in CRC cell lines compared with FHC cells (Fig. 4C). Moreover, the protein levels of TCF7 were also upregulated in CRC tissues and cell lines compared to normal controls (Fig. 4D and E). Through RT-qPCR, we also demonstrated that the expression 
of miR-6852 was negatively correlated with that of TCF7 in CRC tissues (Fig. 4F).

Knockdown of TCF7 inhibits the proliferation and invasion of CRC cells. To further determine the role of TCF7 in CRC cells, we knocked down TCF7 in HCT8 and SW480 with specific siRNA. Western blot analysis indicated that TCF7 was significantly downregulated in HCT8 and SW480 cells transduced with siTCF7 compared to control group (Fig. 5A). CCK-8 assay indicated that knockdown of TCF7 significantly suppressed the proliferation of HCT8 and SW480 cells (Fig. 5B). Moreover, less cells transduced with siTCF7 entered into $\mathrm{S}$ phase (Fig. 5C). Finally, we showed that knockdown of TCF7 also inhibited the invasion of HCT8 and SW480 cells (Fig. 5D). Taken together, our study demonstrated that miR-6852 suppressed the proliferation and invasion of CRC cells by targeting TCF7, which acted as an oncogene in CRC cells.

\section{Discussion}

$\mathrm{CRC}$ is one of the most common malignances and its incidence is increasing year by year (1). However, no effective therapy has been developed for treatment of CRC patients especially with metastasis. The underlying mechanism of CRC progression remains elusive. There is a very urgent requirement to search novel biomarkers for CRC diagnosis or prognosis and therapeutic targets for CRC intervention. Several miRNAs have been demonstrated to be promising indicators of cancer characteristics, including in CRC. Thus, understanding the roles and functional mechanism of miRNAs in CRC progression will contribute to the discovery of biomarkers and therapeutic targets. In this study, we studied a functionally undefined miRNA, miR-6852, in CRC. We also demonstrated its functional mechanism by several experiments.

miRNAs participate in all kinds of biological processes because of their regulatory roles on gene expression (21). Several miRNAs are demonstrated dysregulated in cancers, including gastric cancer (22), colon cancer (23), breast cancer (24), non-small cell lung cancer (25), bladder cancer (26), ovarian cancer (27), prostate cancer (28) and pancreatic cancer (29). In CRC, many miRNAs has been shown to regulate tumor progression. For example, miR-21 post-transcriptionally downregulates tumor suppressor Pdcd 4 and stimulates invasion, intravasation and metastasis in CRC (30). miRNA-27b targets vascular endothelial growth factor $\mathrm{C}$ to inhibit tumor progression and angiogenesis in CRC (31). MicroRNA-497 targets insulin-like growth factor 1 receptor and has a tumor suppressive role in human CRC (32). Previous study indicated miR-6852 induces necrosis in cervical cancer cells (19). However, the function of miR-6852 in CRC remains largely unknown. In this study, we found that miR-6852 was downregulated in CRC tissues and cell lines. Moreover, we found that miR-6852 expression level was negatively correlated with tumor metastasis and positively correlated with patients' survival rate, which suggested that miR-6852 might serve as a prognostic biomarker in CRC. We also demonstrated that overexpression of miR-6852 significantly inhibited the proliferation and invasion of CRC cells. These data suggested that miR-6852 functions as a tumor suppressor in CRC.
TCF7 is well known for its function in T lymphocyte development (33). Previous study indicated that TCF7 can initiate the Wnt/ $\beta$-catenin signaling cascade (34), which is abnormally activated in most CRC cases. Increasing evidences demonstrate TCF7 is widely involved in tumorigenesis, including prostate cancer (35), osteosarcoma (36) and so on. How TCF7 expression is regulated requires to be further investigated. In our study, we found that TCF7 was a target gene of miR-6852 in CRC cells. Overexpression of miR-6852 significantly reduced the mRNA and protein levels of TCF7 in CRC cells. We showed that there was an inverse correlation between the expression of miR-6852 and TCF7 in CRC tissues. Moreover, we found that TCF7 was significantly upregulated in CRC tissues compared with normal tissues. And the expression of TCF7 was correlated with CRC metastasis. Through functional experiments, we demonstrated that TCF7 knockdown significantly suppressed the proliferation and invasion of CRC cells, which indicated that miR-6852-mediated inhibition on cell proliferation and invasion relies on downregulation of TCF7 at least in part.

In conclusion, our study demonstrated that miR-6852 suppressed the proliferation and invasion by targeting TCF7 in CRC cells. And we found that miR-6852 might serve as a prognostic biomarker for CRC patients.

\section{Acknowledgements}

Not applicable.

\section{Funding}

No funding was received.

\section{Availability of data and materials}

All data generated or analyzed during this study are included in this published article.

\section{Authors' contributions}

BC and XH initiated, designed this work, analyzed, interpreted the results and wrote this manuscript. All authors read and approved the final manuscript.

\section{Ethics approval and consent to participate}

For the use of human samples, the protocol for this study was approved by the Institutional Ethics Committee of Harbin Medical University Cancer Hospital and all enrolled patients signed a written informed consent document.

\section{Patient consent for publication}

All patients within this study provide consent for the publication of their data.

\section{Competing interests}

The authors declare that they have no competing interests. 


\section{References}

1. Harrison $\mathrm{S}$ and Benziger $\mathrm{H}$ : The molecular biology of colorectal carcinoma and its implications: A review. Surgeon 9: 200-210, 2011.

2. Song M and Chan AT: Diet, gut microbiota, and colorectal cancer prevention: A review of potential mechanisms and promising targets for future research. Curr Colorectal Cancer Rep 13: 429-439, 2017

3. Powell SM, Zilz N, Beazer-Barclay Y, Bryan TM, Hamilton SR, Thibodeau SN, Vogelstein B and Kinzler KW: APC mutations occur early during colorectal tumorigenesis. Nature 359: 235-237, 1992.

4. Coppedè F, Lopomo A, Spisni R and Migliore L: Genetic and epigenetic biomarkers for diagnosis, prognosis and treatment of colorectal cancer. World J Gastroenterol 20 : 943-956, 2014.

5. Lee YC, Lee YL, Chuang JP and Lee JC: Differences in survival between colon and rectal cancer from SEER data. PLoS One 8 e78709, 2013

6. Ambros V: The functions of animal microRNAs. Nature 431: 350-355, 2004

7. Valencia-Sanchez MA, Liu J, Hannon GJ and Parker R: Control of translation and mRNA degradation by miRNAs and siRNAs. Genes Dev 20: 515-524, 2006.

8. Miranda KC, Huynh T, Tay Y, Ang YS, Tam WL, Thomson AM, Lim B and Rigoutsos I: A pattern-based method for the identification of MicroRNA binding sites and their corresponding heteroduplexes. Cell 126: 1203-1217, 2006.

9. Bartel DP: MicroRNAs: Genomics, biogenesis, mechanism, and function. Cell 116: 281-297, 2004

10. He L and Hannon GJ: MicroRNAs: Small RNAs with a big role in gene regulation. Nat Rev Genet 5: 522-531, 2004.

11. Agrawal L, Sahu S, Ghosh S, Shiga T, Fujita D and Bandyopadhyay A: Inventing atomic resolution scanning dielectric microscopy to see a single protein complex operation live at resonance in a neuron without touching or adulterating the cell. J Integr Neurosci 15: 435-462, 2016.

12. Zhang B, Pan X, Cobb GP and Anderson TA: microRNAs as oncogenes and tumor suppressors. Dev Biol 302: 1-12, 2007.

13. Lu G, Fu D, Jia C, Chai L, Han Y, Liu J, Wu T, Xie R, Chang Z, Yang H, et al: Reduced miR-105-1 levels are associated with poor survival of patients with non-small cell lung cancer. Oncol Lett 14: 7842-7848, 2017.

14. Tahiri A, Aure MR and Kristensen VN: MicroRNA networks in breast cancer cells. Methods Mol Biol 1711: 55-81, 2018.

15. Du X, Liu B, Luan X, Cui Q and Li L: miR-30 decreases multidrug resistance in human gastric cancer cells by modulating cel autophagy. Exp Ther Med 15: 599-605, 2018.

16. Dopeso H, Rodrigues P, Bilic J, Bazzocco S, Cartón-García F, Macaya I, de Marcondes PG, Anguita E, Masanas M, Jiménez-Flores LM, et al: Mechanisms of inactivation of the tumour suppressor gene RHOA in colorectal cancer. $\mathrm{Br}$ J Cancer 118: 106-116, 2018.

17. Cao Q, Liu F, Ji K, Liu N, He Y, Zhang W and Wang L: MicroRNA-381 inhibits the metastasis of gastric cancer by targeting TMEM16A expression. J Exp Clin Cancer Res 36: 29 , 2017.

18. Shi ZM, Wang L, Shen H, Jiang CF, Ge X, Li DM, Wen YY, Sun HR, Pan MH, Li W, et al: Downregulation of miR-218 contributes to epithelial-mesenchymal transition and tumor metastasis in lung cancer by targeting Slug/ZEB2 signaling. Oncogene 36: 2577-2588, 2017

19. Poudyal D, Herman A, Adelsberger JW, Yang J, Hu X, Chen Q, Bosche M, Sherman BT and Imamichi T: A novel microRNA, hsa-miR-6852 differentially regulated by Interleukin-27 induces necrosis in cervical cancer cells by downregulating the FoxM1 expression. Sci Rep 8: 900, 2018

20. Livak KJ and Schmittgen TD: Analysis of relative gene expression data using real-time quantitative PCR and the 2(-Delta Delta C(T)) method. Methods 25: 402-408, 2001.

21. Garzon R, Fabbri M, Cimmino A, Calin GA and Croce CM MicroRNA expression and function in cancer. Trends Mol Med 12: 580-587, 2006
22. Guo J, Miao Y, Xiao B, Huan R, Jiang Z, Meng D and Wang Y: Differential expression of microRNA species in human gastric cancer versus non-tumorous tissues. J Gastroenterol Hepatol 24 652-657, 2009.

23. Díaz R, Silva J, García JM, Lorenzo Y, García V, Peña C, Rodríguez R, Muñoz C, García F, Bonilla F and Domínguez G: Deregulated expression of miR-106a predicts survival in human colon cancer patients. Genes Chromosomes Cancer 47: 794-802, 2008.

24. Wang Y, Yu Y, Tsuyada A, Ren X, Wu X, Stubblefield K, Rankin-Gee EK and Wang SE: Transforming growth factor- $\beta$ regulates the sphere-initiating stem cell-like feature in breast cancer through miRNA-181 and ATM. Oncogene 30: 1470-1480, 2011.

25. Li J, Yang H, Li Y, Liu Y, Chen S, Qi C, Zhang Q, Lan T, He X, Guan XY and Wang L: microRNA-146 up-regulation predicts the prognosis of non-small cell lung cancer by miRNA in situ hybridization. Exp Mol Pathol 96: 195-199, 2014.

26. Canturk KM, Ozdemir M, Can C, Öner S, Emre R, Aslan H, Cilingir O, Ciftci E, Celayir FM, Aldemir O, et al: Investigation of key miRNAs and target genes in bladder cancer using miRNA profiling and bioinformatic tools. Mol Biol Rep 41: 8127-8135, 2014.

27. Yang H, Kong W, He L, Zhao JJ, O'Donnell JD, Wang J, Wenham RM, Coppola D, Kruk PA, Nicosia SV and Cheng JQ: MicroRNA expression profiling in human ovarian cancer: miR-214 induces cell survival and cisplatin resistance by targeting PTEN. Cancer Res 68: 425-433, 2008.

28. Beltran H, Yelensky R, Frampton GM, Park K, Downing SR, MacDonald TY, Jarosz M, Lipson D, Tagawa ST, Nanus DM, et al: Targeted next-generation sequencing of advanced prostate cancer identifies potential therapeutic targets and disease heterogeneity. Eur Urol 63: 920-926, 2013

29. Dillhoff M, Liu J, Frankel W, Croce C and Bloomston M: MicroRNA-21 is overexpressed in pancreatic cancer and a potential predictor of survival. J Gastrointest Surg 12: 2171-2176, 2008.

30. Asangani IA, Rasheed SAK, Nikolova DA, Leupold JH, Colburn NH, Post S and Allgayer H: MicroRNA-21 (miR-21) post-transcriptionally downregulates tumor suppressor Pdcd4 and stimulates invasion, intravasation and metastasis in colorectal cancer. Oncogene 27: 2128-2136, 2008.

31. Ye J, Wu X, Wu D, Wu P, Ni C, Zhang Z, Chen Z, Qiu F, Xu J and Huang J: miRNA-27b targets vascular endothelial growth factor $\mathrm{C}$ to inhibit tumor progression and angiogenesis in colorectal cancer. PLoS One 8: e60687, 2013.

32. Guo ST, Jiang CC, Wang GP, Li YP, Wang CY, Guo XY, Yang RH, Feng Y, Wang FH, Tseng HY, et al: MicroRNA-497 targets insulin-like growth factor 1 receptor and has a tumour suppressive role in human colorectal cancer. Oncogene 32: 1910-1920, 2013

33. Weber BN, Chi AW, Chavez A, Yashiro-Ohtani Y, Yang Q, Shestova $\mathrm{O}$ and Bhandoola A: A critical role for TCF-1 in T-lineage specification and differentiation. Nature 476: 63-68, 2011.

34. Cui L, Guan Y, Qu Z, Zhang J, Liao B, Ma B, Qian J, Li D, Li W, $\mathrm{Xu}$ GT and Jin Y: WNT signaling determines tumorigenicity and function of ESC-derived retinal progenitors. J Clin Invest 123: 1647-1661, 2013

35. Chen WY, Liu SY, Chang YS, Yin JJ, Yeh HL, Mouhieddine TH, Hadadeh O, Abou-Kheir W and Liu YN: MicroRNA-34a regulates WNT/TCF7 signaling and inhibits bone metastasis in Ras-activated prostate cancer. Oncotarget 6: 441-457, 2015.

36. Wang Y,Zhang S, Xu Y,Zhang Y, Guan H,Li X, Li Y and Wang Y: Upregulation of miR-192 inhibits cell growth and invasion and induces cell apoptosis by targeting TCF7 in human osteosarcoma. Tumor Biol 37: 15211-15220, 2016.

This work is licensed under a Creative Commons Attribution-NonCommercial-NoDerivatives 4.0 International (CC BY-NC-ND 4.0) License. 